\title{
Iatrogenic Dissection of The Right Coronary Artery and The Ascending Aorta Secondary to Percutaneous Coronary Intervention: A Case Report
}

\author{
Changjiang Yu*, Fan He*, Huaidong Chen, Jianmao Hong, Ximing Qian \\ Department of Cardiac Surgery, Sir Run Run Shaw Hospital, School of Medicine, Zhejiang University, Hangzhou, China
}

\section{ABSTRACT}

Iatrogenic dissections of coronary artery and aorta is a rare and potentially fatal event, often following percutaneous coronary intervention (PCI). The prognosis of patients often depends on early accurate diagnosis and timely and effective treatment. There are no definite guidelines for the treatment of acute coronary artery dissection caused by PCI. Here, we report a 50-year-old patient with iatrogenic dissection of the right coronary artery and type A aortic dissection who underwent PCI for chest pain. We performed emergency surgery of right coronary artery ostium repair, aortic valvoplasty, ascending aorta replacement, and aortic arch descending stent implantation for the patient. The operation went smoothly, and the patient successfully was discharged two weeks after the operation.

\section{INTRODUCTION}

With the increase of PCI in cardiology, catheter-related complications also have increased. However, coronary artery dissection and acute aortic dissection due to PCI are rare and fatal [Moles 1992]. Several proposed mechanisms have been described in literature to explain the etiology of this fatal event, including retrograde spread of a coronary artery dissection as a result of mechanical trauma by instruments used during the procedure, such as a guide catheter, wire, inflated balloon, etc. [Shah 2016]. At present, there are no clear treatment plans for coronary artery dissection and acute aortic dissection caused by PCI.

The purpose of this paper is to present a clinical case of iatrogenic right coronary artery dissection due to PCI that extends to acute type $\mathrm{A}$ aortic dissection of the ascending aorta and to share our experience in the treatment.

Received March 29, 2020; received in revised form May 5, 2020; accepted May 6, 2020 .

*These authors contributed equally to this work.

Correspondence: Ximing Qian, Department of Cardiac Surgery, Sir Run Run Shaw Hospital, School of Medicine, Zhejiang University, Hangzhou, China (e-mail:3310005@zju.edu.cn).

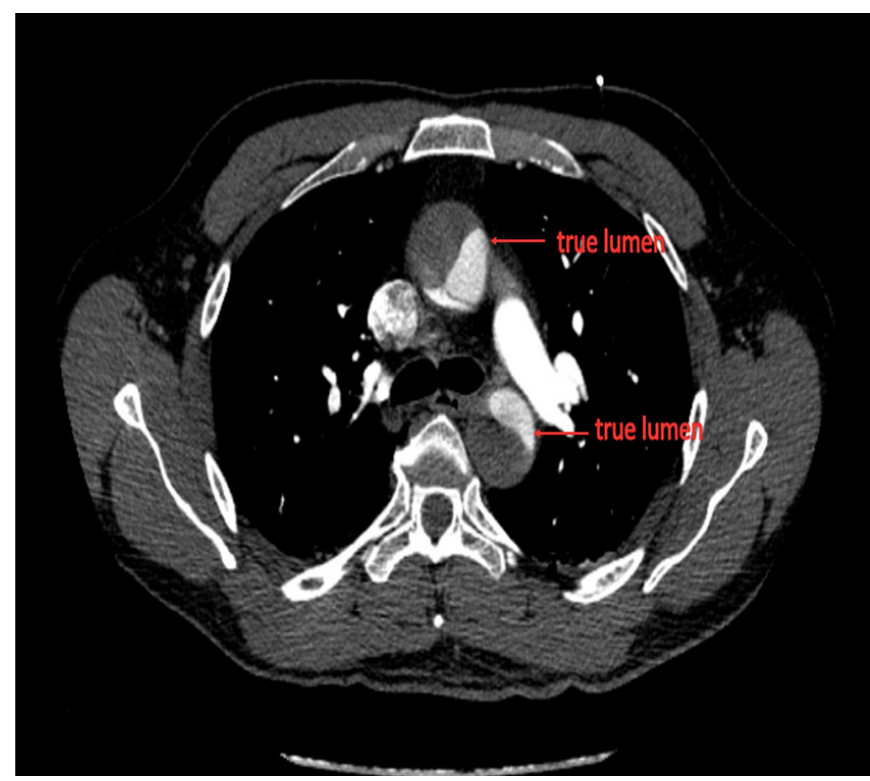

Figure 1. Aortic dissection developed from the aortic root to the descending aorta with a stenosed true lumen.

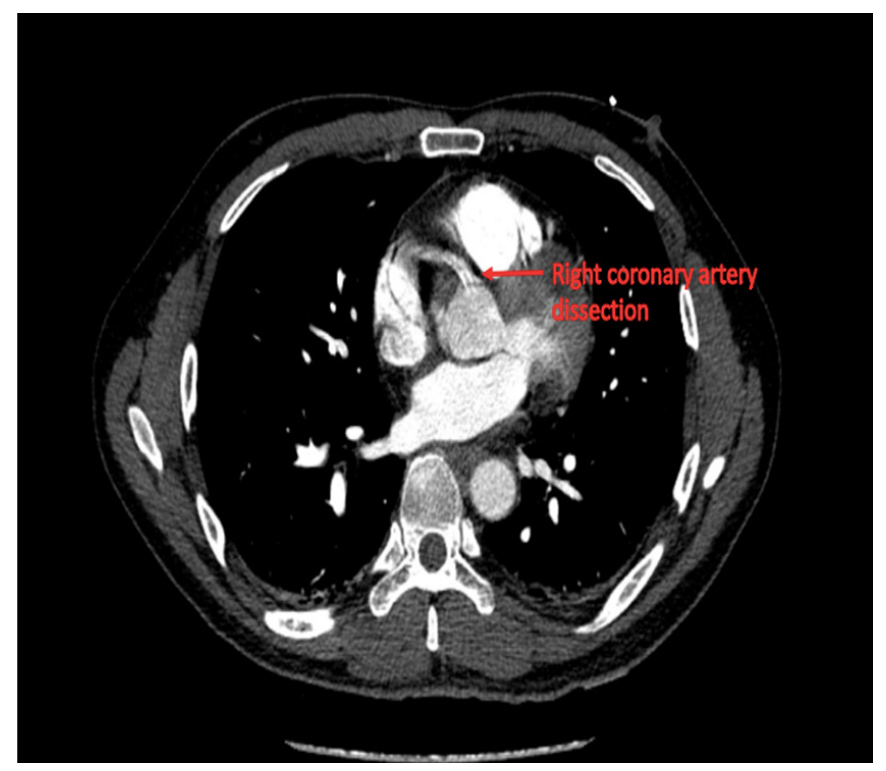

Figure 2. The image showed the coronary artery dissection at the opening of the right coronary artery. 


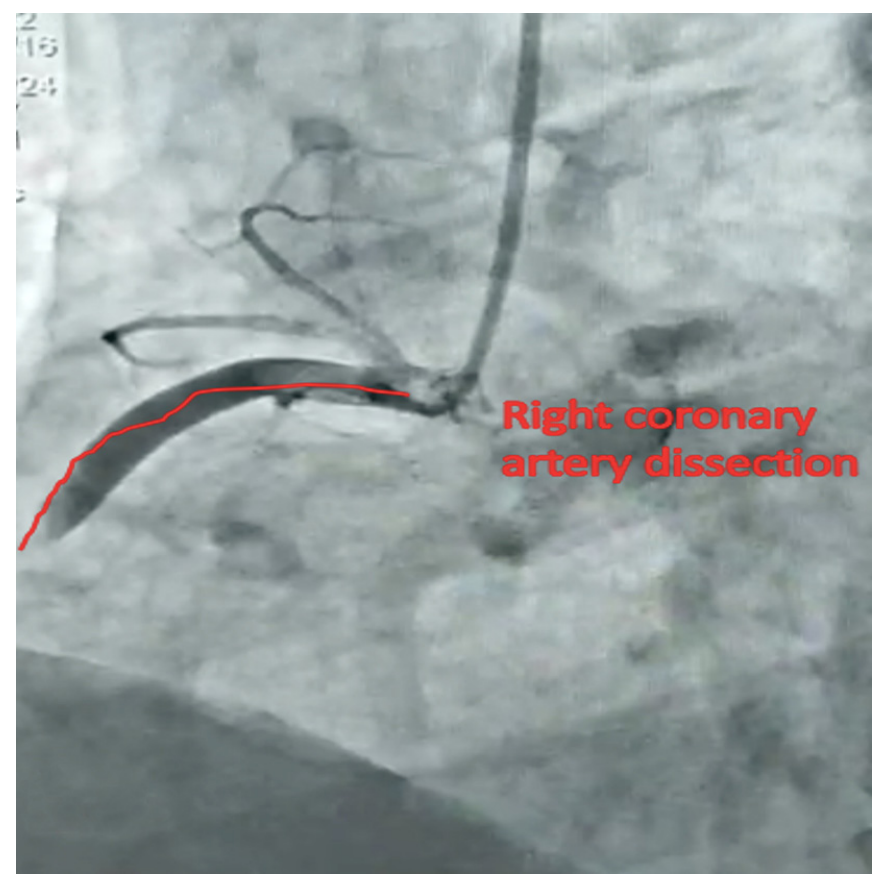

Figure 3. Coronary angiography in local hospital confirmed the coronary artery dissection at the opening of the right coronary artery.

\section{CASE REPORT}

A 50-year-old male patient underwent coronary angiography in the local hospital because of severe chest pain for two days. During the angiography, a right coronary artery dissection was found, due to violent manipulation of the guide catheter. But the cardiologist mistakenly believed that the right coronary artery was occluded, so a bare metal stent was inserted at the right coronary artery dissection. As a result, the acute type A aortic dissection was developed. Subsequently, the patient was transferred to our hospital. The patient had a history of hypertension for five years and did not regularly take medication. He had a 5-year history of laser lithotripsy for kidney stones and replantation of the severed little finger of his left hand. His vital signs were stable after admission. Physical examination indicated that the breath sounds in both lungs were coarse without obvious rales, and the first heart sound was weakened without obvious murmurs. Examination results showed that NT proBNP was $1599 \mathrm{pg} / \mathrm{mL}$, AST $120 \mathrm{U} / \mathrm{L}, \mathrm{WBC} 16.5 \times 10^{9} / \mathrm{L}$, Troponin I $13.4 \mathrm{ng} / \mathrm{mL}$ and myocardial enzyme spectrum significantly were increased. Aortic enhanced CT suggested that the dissection developed from the aortic root to the descending aorta with a stenosed true lumen (Figure 1) and a dissection at the right coronary artery ostium (Figure 2). The echocardiography indicated a mild aortic valve regurgitation and acute inferior wall myocardial infarction was found through the electrocardiogram. Of course, coronary angiography from the local hospital also confirmed the dissection of the right coronary artery opening (Figure 3).

We performed an emergency surgery of aortic dissection repair. The operation adopted the median sternal incision and

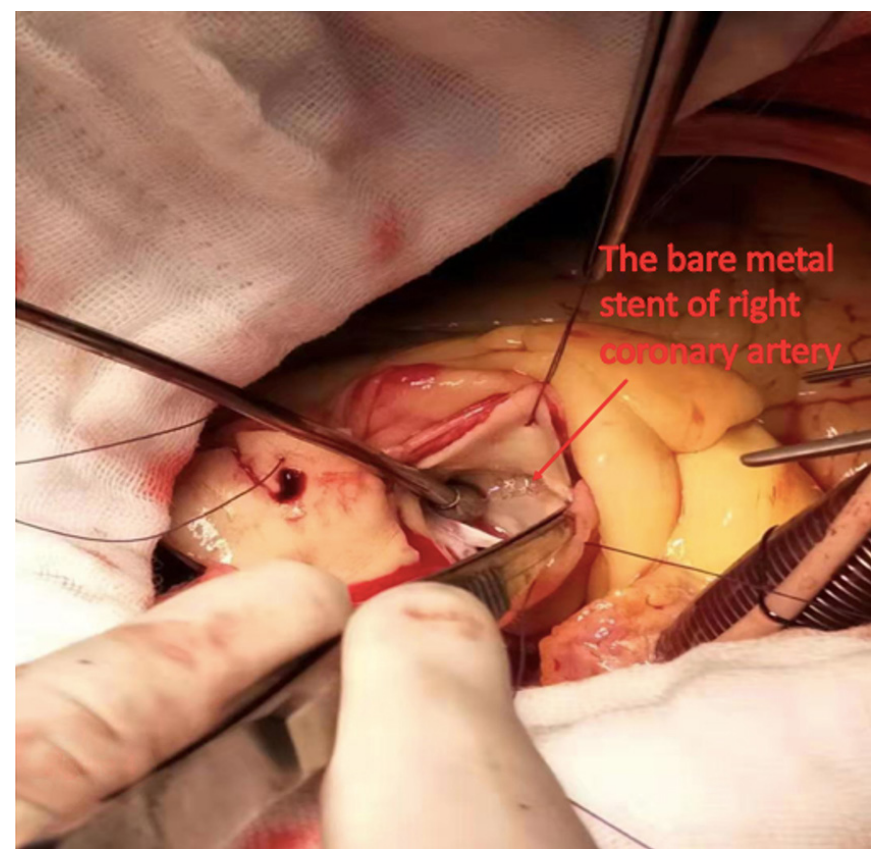

Figure 4. An inserted bare-metal stent was found in the false lumen of right coronary dissection.

extracorporeal circulation was established by bidirectional arterial blood supply of the right femoral artery and axillary artery. We found an enlargement of the left ventricle and a widened ascending aorta about $4.5 \mathrm{~cm}$ in diameter, with thin tunica adventitia in some segments. The tear of the aortic dissection was located above the right coronary artery opening, and the dissection was performed along the proximal part of the aorta to the distal part to the descending aorta. The baremetal stent was seen at the right coronary artery opening, which extended into the false lumen (Figure 4). The aortic valve had a poor involution. We removed the coronary artery stent and repaired the right coronary artery opening with a 6-0 prolene suture and artificial vascular patch. The junctions of aortic valve were suspended, and valvuloplasty was performed. The ascending aorta was excised, a 26\# Medtronic artificial vessel was replaced by the "sandwich method," and at the same time, a 26\# trunk stent was placed at the distal end of aortic arch through circulatory arrest. The stent at the openings of the three branches were fenestrated and fixed with a 4-0 prolene suture and felt patches by unilateral cerebral perfusion through the axillary artery at a temperature of $25^{\circ} \mathrm{C}$. The purpose of this method was to prevent the displacement of the trunk stent and the internal leakage of the distal end of the left subclavian artery. The patient underwent a successful operation, the cardiopulmonary bypass time was 108 minutes, and the cross-clamp time 83 minutes. Postoperative aortic enhanced CT indicated that the three branches of the aorta and the right coronary artery were well developed (Figure 5). The liver function, myocardial enzyme spectrum, and troponin I were all normal. The patient was discharged two weeks after the operation. 


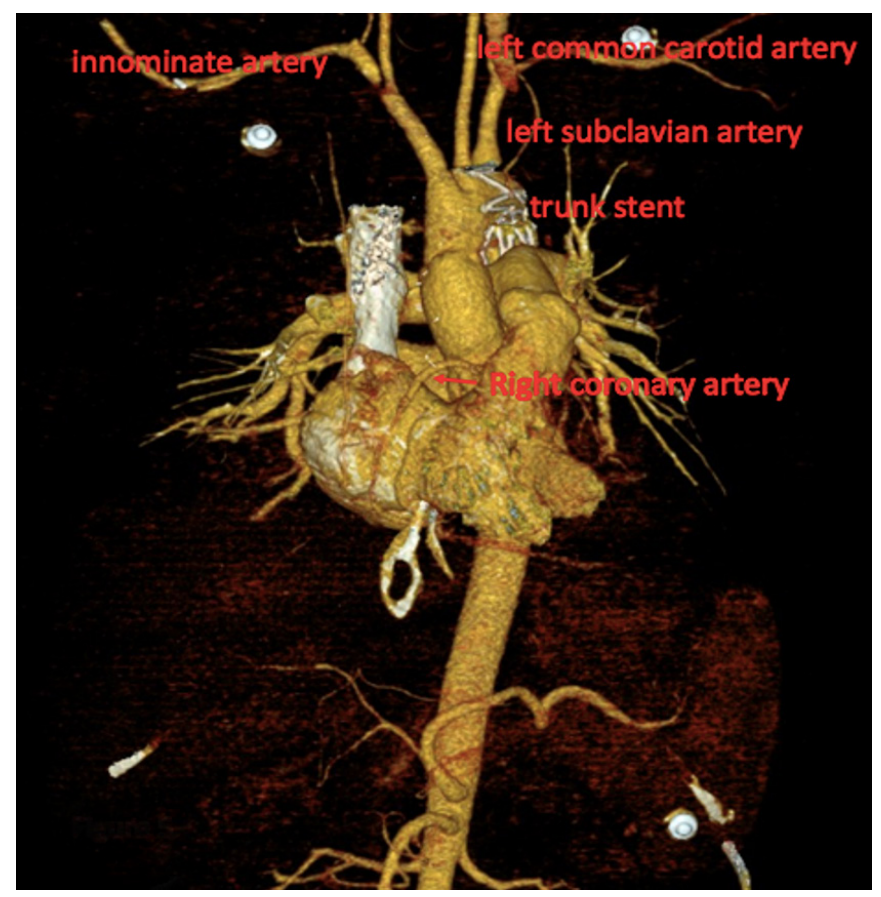

Figure 5. The postoperative CT examination results suggested the three branches of the aorta and the right coronary artery were well developed. The stent of descending aorta was in good position.

\section{DISCUSSION}

Acute aortic dissection during PCI remains a very rare complication, with an overall incidence of $0.02 \%$ [Wyss 2008]. Most of the previous studies reported that aortic dissection rupture originated in the coronary artery dissection, and however, studies of pure ascending aortic dissection without involving the coronary arteries were rarely reported [Mauser 1999]. It has been mentioned that the iatrogenic aortocoronary dissection involved the RCA ostium in about $85 \%$ of the published cases, most commonly during PCI of chronic total occlusion (CTO) [Carstensen 2008]. Several proposed mechanisms have been described in literature to explain the etiology of this fatal event, including mechanical trauma by the catheter tip during the procedure, over inflation and dilation of the balloon or stent, the dilation of a calcified plaque, the fracture of the stent strut or from aggressive handling of guide wires [Dahdouh 2012].

For our case, the formation of the right coronary artery dissection may be due to the improper operation of the guide catheter during coronary angiography, resulting in direct mechanical trauma to the right coronary artery orifice by the catheter tip. However, the implantation of the coronary stent into the right coronary dissection improperly and the injection of a large amount of contrast agent rapidly aggravated the dissection to the distal coronary artery in the anterograde direction and the aorta in the opposite direction.

The appropriate therapeutic strategy for iatrogenic dissections remains controversial being generally based on the type of dissection and the hemodynamic stability of the patient. However, no guidelines have yet been established to address this point specially [El-Haress 2017]. Considering the treatment of such cases, iatrogenic coronary artery dissections are classified into three forms by Dunning et al, where class I denotes dissection involving only the coronaries, class II extending up to $<40 \mathrm{~mm}$ of the ascending aorta, and class III reaching $>40 \mathrm{~mm}$ of the ascending aorta [Dunning 2000]. As class I and II patients with limited involvement of the aorta can benefit from stenting of the coronary dissection entry point without the need for surgical intervention, it was found that urgent surgery is the treatment of choice for class III patients with extensive dissection or patients with hemodynamic instability and those with ischemia of one of the aortic branches [El-Haress 2017]. Of course, surgery also can be beneficial in patients with severe aortic regurgitation, due to dissection involving the aortic valve. For our patient, the involvement of the coronary dissection into the descending aorta clearly was classified as class III. And because the bare-metal stent was inserted into the false lumen of the right coronary dissection, emergency surgical intervention was the first choice for our patient. Since distal flow of the right coronary artery through intraoperative assessment was acceptable, we just removed the bare-metal stent and repaired the opening of the right coronary artery dissection with an artificial vascular patch, thus achieving complete isolation of the coronary dissection.

Since the aortic dissection of the patient was type I, involving the aortic arch and descending aorta, we performed a surgery of ascending aorta replacement, aortic arch shaping, and descending aorta stent placement. However, the three branches of the aortic arch were not involved, so we chose the fenestration technique for the treatment of the three branches. In particular, we used 4-0 propene sutures and felt patches for hemlock fixation at all the windowing sites along the long axis of aortic arch, so as to prevent the displacement of the trunk stent and reduce the incidence of reoperation, due to type I internal leakage postoperation. Of course, our patients recovered very well, and the postoperative enhanced CT also showed good angiography of the right coronary artery and the three branches, with no internal leakage.

\section{CONCLUSIONS}

Iatrogenic aortic coronary dissection is a rare and feared complication for cardiologists and can lead to fatal consequences if not promptly treated. For acute type A aortic dissection caused by coronary dissection, urgent surgical repair is helpful to the patient's prognosis and should be the primary treatment. Some surgical techniques should be mastered to avoid postoperative complications.

\section{ACKNOWLEDGMENTS}

This work was supported by a grant from The National Key Research and Development Program of China (no. 2017YFC1308004).) 


\section{REFERENCES}

Carstensen S, Ward MR. 2008. Iatrogenic aortocoronary dissection: The case for immediate aortoostial stenting. Heart Lung Circ 17:325-329.

Dahdouh Z, Roule V, Lognoné T, et al. 2012. Iatrogenic bidirectional dissection of the right coronary artery and the ascending aorta: the worst nightmare for an interventional cardiologist. [J]. Korean Circ J 42: 504-6.

Dunning DW, Kahn JK, Hawkins ET, et al. 2000. Iatrogenic coronary artery dissections extending into and involving the aortic root. [J].Catheter Cardiovasc Interv 51: 387-93.

El-Haress M, Daadaa H, Shahjouei S, et al. 2017. Iatrogenic Acute Ascending Aortic Dissection with Intramural Hematoma during Coronary Artery Stenting: A Case Report. [J]. Front Surg 4: 2.
Mauser M, Ennker J, Fleischmann D. 1999. Dissection of the sinus valsalvae aortae as a complication of coronary angioplasty. Z Kardiol $88: 1023-7$.

Moles VP, Chappuis F, Simonet F, Urban P, De La Serna F, Pande AK, et al. 1992. Aortic dissection as complication of percutaneous transluminal coronary angioplasty. Cathet Cardiovasc Diagn 1992 26:8-11.

Shah P, Bajaj S, Shamoon F. 2016. Aortic dissection caused by percutaneous coronary intervention: 2 new case reports and detailed analysis of 86 previous cases. Tex Heart Inst J 43:52-60.

Wyss CA, Ste el J, Luescher TF. 2008. Isolated acute iatrogenic aortic dissection during percutaneous coronary intervention without involvement of the coronary arteries. J Invasive Cardiol 20:380-2. 